\title{
Middle mediastinal thymic carcinoma with cystic findings in radiologic images: a case report
}

\author{
Shuichi Shinohara*, Koji Kuroda, Taiji Kuwata, Masaru Takenaka, Soichi Oka, Yasuhiro Chikaishi, Ayako Hirai, \\ Naoko Imanishi and Fumihiro Tanaka
}

\begin{abstract}
Background: Thymic carcinomas are usually detected in the anterior mediastinum. Thymic carcinoma occurring in the middle mediastinum and showing an image of a cyst is extremely rare.

Case presentation: A 64-year-old man with a middle mediastinal tumor detected incidentally by a CT scan was referred. Chest $C T$ showed an entirely cystic mass in the middle mediastinum between the bilateral brachiocephalic vein and trachea. We resected the tumor completely in the right recurrent nerve because it had invaded the nerve. Immunohistochemical features showed a thymic carcinoma pattern. The final diagnosis was thymic squamous cell carcinoma occurring in the middle mediastinum.
\end{abstract}

Conclusion: To our best knowledge, this is the first report of a thymic carcinoma occurring in the middle mediastinum, as demonstrated by histopathological findings with immunohistochemical features.

Keywords: Cyst, Middle mediastinum, MRI, Thymic carcinoma

\section{Background}

Thymic neoplasms usually are detected in the anterior mediastinum. Fewer than $4 \%$ of thymic tumors occur in the middle mediastinum, defined as mediastinal space surrounded by the left brachiocephalic vein, superior vena cava, esophagus, trachea, and main bronchus up to the cross section of the left brachiocephalic vein and the center of the trachea by the Japanese Association for Research on the Thymus [1]. Thymic carcinoma is a rare thymic neoplasm. Moreover, there are few reports of middle mediastinal thymic carcinomas histopathologically diagnosed as having immunohistochemical features. We report a rare case of thymic carcinoma in the middle mediastinum that had cystic findings on computed tomography (CT) and magnetic resonance imaging (MRI).

\section{Case presentation}

A 64-year-old man was referred to our hospital for a middle mediastinal tumor detected incidentally by a CT scan. Chest CT showed an entirely cystic mass with a thick capsule slightly enhanced in the middle mediastinum between the bilateral brachiocephalic vein and trachea (Fig. 1a). At CT scan, the thymus is of normal size and located in the anterior mediastinum as a lowdensity triangle area. The mass had no solid component. T2-weighted MRI revealed that the main tumor had a heterogeneous isodense signal intensity and that the tumor was encapsulated by a low-signal area (Fig. 1b). There was no gadolinium-enhanced area in this tumor. This radiologic finding indicated the possibility of the mass being a hemorrhagic cyst, bronchogenic cyst, neurogenic tumor, or teratoma, with a small proportion of fat component.

The patient was placed in the left lateral decubitus position, and a right thoracic approach with three-port video-assisted thoracoscopic surgery (VATS) was performed. The tumor was surrounded by the trachea, right main bronchus, brachiocephalic artery, superior vena

\footnotetext{
Second Department of Surgery, School of Medicine, University of Occupational and Environmental Health, 1-1 Iseigaoka, Yahatanishi-ku, Kitakyushu 807-8555, Japan
} 


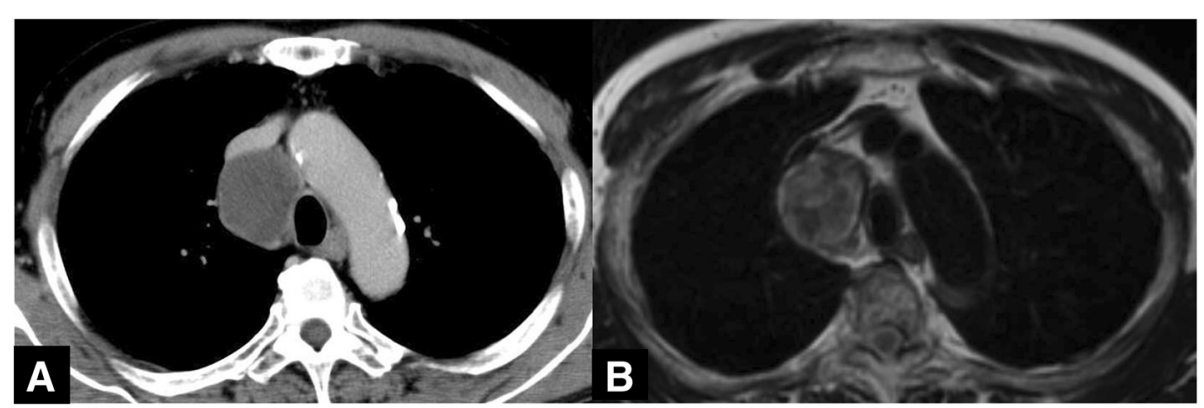

Fig. 1 a Chest CT scan shows a low-density mass in the middle mediastinum surrounded by the brachiocephalic vein, brachiocephalic artery, and trachea. b T2-weighted MRI reveals the main tumor to have a heterogeneous isodense signal intensity, and the tumor was encapsulated by a low-signal area

cava (SVC), and left brachiocephalic vein. It was greatly adherent to the lateral trachea, right main bronchus, and recurrent nerve. Due to the complicated adhesiolysis in the anterior brachiocephalic artery and tumor involving the recurrent nerve, we decided to change the procedure to open thoracotomy. The tumor and recurrent nerve whose length was about $10 \mathrm{~mm}$ were removed while keeping them encapsulated. The tumor measured $45 \times 35 \times 30 \mathrm{~mm}$ and contained red-brown necrotic tissue surrounded by a fibrous capsule. Cross-sectional slices showed a small solid component $(8 \mathrm{~mm})$. The tumor nodule existed along a fibrous capsule multiply (Fig. 2a).

Microscopic findings showed a proliferation of markedly atypical polygonal epithelial cells having hyperchromatic nuclei associated with extensively necrotic and hemorrhagic areas (Fig. 2b, c). The recurrent nerve was involved with the carcinoma cells. Immunohistochemically, the carcinoma cells were positive for AE1/AE3, CAM5.2, p40, p63, CK34betaE12, CD5, and bcl-2 but negative for CK5/6, TTF-1, c-kit, AFP, and CD30. This feature indicated poorly differentiated, thymic squamous cell carcinoma in pathological T3NOM0 stage III. The margin of the resected tumor was free of disease.

Adjuvant concurrent chemoradiotherapy was performed. We administered monthly carboplatin plus paclitaxel for four courses, and the radiation therapy dose was $50 \mathrm{~Gy}$. There was no recurrence 6 months after surgery.

\section{Conclusions}

To our best knowledge, this is the first report of a thymic carcinoma occurring in the middle mediastinum, as demonstrated by histopathological findings with immunohistochemical features. Moreover, radiological findings demonstrating a cyst with no solid component in the middle mediastinum made preoperative diagnosis of a thymic carcinoma difficult.

Thymic carcinoma is an uncommon neoplasm and occurs in $5.5 \%$ of all resected mediastinal tumors [2]. Furthermore, a middle mediastinal thymic carcinoma is extremely rare. Thymic carcinoma occurs in the anterior mediastinum, and ectopic thymic carcinomas, which are carcinomas that show thymus-like differentiation, are rare [3]. Ectopic thymic carcinoma is reported in cases of intrathyroid neoplasms [3], even though the first case reported was that of a middle mediastinal thymic carcinoma with histopathologic features. We think ectopic thymus tissue existed in middle mediastinum and it became a progressive neoplasm.

Thymic carcinoma is difficult to diagnose microscopically, whereas immunohistochemical features are greatly

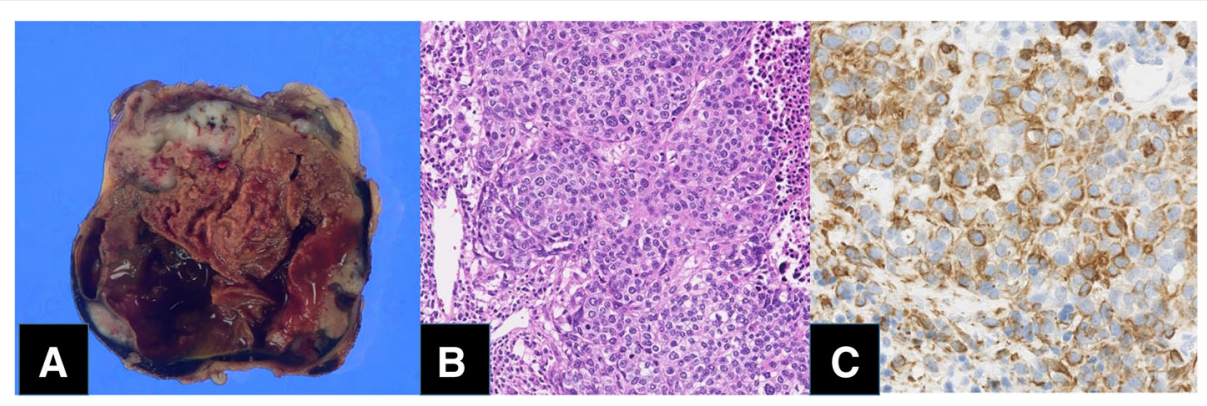

Fig. 2 a Macroscopic findings of cross-sectional slices showed a small solid component (8 mm). b Microscopy revealed a proliferation of markedly atypical polygonal epithelial cells having hyperchromatic nuclei (×400). c Immunohistochemically, tumor cells are positive for CD5 (×400) 
useful to make an exact diagnosis. CD5 is specific to the thymic epithelial cell, and metastatic tumor is excluded [4, 5]. To distinguish thymic squamous cell carcinoma from pulmonary squamous cell carcinoma is quite difficult. However, CD5 positive is useful for accurate diagnosis because pulmonary squamous cell carcinoma is consistently negative for CD5 by a large study of 1465 non-small cell lung cancer cases [5]. We diagnosed thymic squamous cell carcinoma by both microscopic findings and immunohistochemical findings. Middle mediastinal thymic carcinoma diagnosed by only microscopic findings was reported [6]. However, the immunohistochemical findings did not indicate thymic carcinoma because it was negative for CD5, which is specific to the thymic epithelial cell, and assessment for other thymic carcinoma markers (p53, c-kit, and bcl-2) was not performed. In our case, the immunohistochemical feature was coincident with thymic squamous cell carcinoma, and the possibility of other metastatic neoplasms in the mediastinum was excluded by imaging tests.

Mediastinal tumors showing cystic findings are generally benign. Malignancy has been reported in $2.4 \%$ of all cases with cystic findings detected by mediastinum CT [7]. Focal cystic change with thymic neoplasm, occurring in $40 \%$ of resected thymomas, is usual because thymic carcinomas and thymomas contain fluid, bleeding, and necrotic tissue with degeneration [8]. When high-grade degeneration developed, the tumor demonstrated cystic findings, such as in this case. Cystic thymomas have occasionally been reported, even though cystic thymic carcinoma is rare [8]. The malignant component in the microscopic findings was too small to be detected by imaging tests, and it made difficult to consider malignant tumor before operation.

Thymic carcinoma can occur in the middle mediastinum. Moreover, when cystic findings are noted in the middle mediastinum, thymic carcinoma should be considered in the differential diagnosis because the operative strategy and therapy of thymic carcinoma differ from those of other benign tumors.

\section{Abbreviations}

CT: Computed tomography; MRI: Magnetic resonance imaging

\section{Acknowledgements}

We are grateful to Dr. Eisuke Shiba and Dr. Takahiko Kasai, belonging to the Department of Pathology and Oncology, School of Medicine, University of Occupational and Environmental Health, for their exact pathological diagnosis.

\section{Funding}

We had no financial assistance of this manuscript.

\section{Authors' contributions}

SS, KK, AH, and FT performed the operation. TK, MT, SO, YC, and NI supported the postoperative management. KK and $\mathrm{FT}$ checked and revised the manuscript. All authors read approved the final version of this manuscript.

\section{Competing interests}

The authors declare that they have no competing interests.

\section{Consent for publication}

Written informed consent was obtained from the patient for publication of this case report and any accompanying images. A copy of the written consent is available for review by the Editor-in-Chief of this journal.

Received: 28 September 2016 Accepted: 2 November 2016

Published online: 07 November 2016

\section{References}

1. Rosai J, Levine GD. Tumors of the thymus. In: Fuminger HI, editor. Atlas of tumor pathology. Fascicle 13, 2nd series. Washington: Armed Forces Institute of Pathology; 1976. p. 34-161.

2. Kuwano H, Amano J, Yokomise $\mathrm{H}$. Thoracic and cardiovascular surgery in Japan during 2010: annual report by The Japanese Association for Thoracic Surgery. Gen Thorac Cardiovasc Surg. 2012;60:680-708.

3. Chan JK, Rosai J. Tumors of the neck showing thymic or related branchial pouch differentiation: a unifying concept. Hum Pathol. 1991;22:349-67.

4. Hishima T, Fukayama M, Fujisawa M, Hayashi Y, Arai K, Funata N, et al. CD5 expression in thymic carcinoma. Am J Pathol. 1994;145:268-75.

5. Kriegsmann M, Muley T, Harms A, Tavernar L, Goldmann T, Dienemann H, et al. Differential diagnostic value of CD5 and CD117 expression in thoracic tumors: a large scale study of 1465 non-small cell lung cancer cases. Diagn Pathol. 2015:10:210.

6. Vernon J, Schieman C, Schneider L, Hanna WC. Rare case of subcarinal thymic carcinoma in the middle mediastinum. J Surg Case Rep. 2015;3:1-2.

7. Hirano S, Motohara T, Nishibe T, Narita Y, Okubo T, Takahashi T, et al. Cystic mediastinal tumor: a clinical study. Nihon Kyoubu Gekagakkai Zasshi. 1997; 11:13-9.

8. Suster S, Rosai J. Cystic thymomas. A clinicopathologic study of ten cases. Cancer. 1992:69:92-7.

\section{Submit your manuscript to a SpringerOpen ${ }^{\circ}$ journal and benefit from:}

- Convenient online submission

- Rigorous peer review

- Immediate publication on acceptance

- Open access: articles freely available online

- High visibility within the field

- Retaining the copyright to your article

Submit your next manuscript at springeropen.com 